\title{
119 HEART AND SOUL: EFFECTIVE ADVANCE CARE PLANNING PROGRAMME PROVIDES INTERNATIONAL MODEL
}

P Malley, ${ }^{1} \mathrm{M}$ Jane Markley, ${ }^{2} \mathrm{~L}$ Rastas ${ }^{3}{ }^{1}$ Aging with Dignity, Florida, USA; ${ }^{2} \mathrm{M}$. Jane Markley Consulting, LLC, Maryland, USA; ${ }^{3}$ Australian Pastoral Health Care Network, Melbourne, Australia

10.1136/bmjspcare-2013-000491.119

Background Aging with Dignity introduced the ACP tool Five Wishes in the USA in 1998 with a focus on personal care, family relationships, dignity, and spirituality. The concept is now helping to shape international efforts. Programme expansion includes Voicing $M y$ Choices: A Planning Guide for Adolescents and Young Adults, developed with the National Institutes of Health. Aim Describe a non-traditional, and replicable, approach to ACP that has proven effective and is positively influencing the development of ACP programmes internationally.

Methods Compilation of data on issues identified as 'most important' at the end-of-life, examination of an ACP resource that responds to these issues, review of data from recent NIH study of perceptions of ACP by adolescents and young adults, and an overview of the influence on international ACP efforts.

Results More than 19 million Five Wishes documents, available in 27 languages, are in circulation. More than 35000 partner organisations include hospitals, health systems, and places of worship. Known sites of replication efforts include Australia, China, Taiwan, Norway, and Italy.

Discussion Having a user-friendly and easy-tounderstand tool that encourages people to discuss their personal, emotional, and spiritual needs along with their medical wishes results in increased completion of usable and meaningful advance care plans.

Conclusion People respond well to ACP when matters of the heart and soul are included in the process, as well as when patients and families are 
empowered to make their own decisions. Lessons learned in this initiative provide helpful guidance for international efforts to encourage ACP. 